\title{
Simulation based optimization of light-outcoupling in organic light-emitting diodes
}

\author{
Stefan Nowy, Jörg Frischeisen, and Wolfgang Brütting \\ Institute of Physics, University of Augsburg, Germany
}

\begin{abstract}
The internal quantum efficiency of organic light-emitting diodes (OLEDs) can reach values close to $100 \%$ if phosphorescent emitters to harvest triplet excitons are used, however, the fraction of light that is actually leaving the device is considerably less. In this work we use numerical simulations to optimize light outcoupling from different OLED stacks. First, we change the distance of the emission zone to the cathode, which minimizes the excitation of surface plasmons. Then the influence of different dipole orientation of the emitter material on the light outcoupling is studied. Finally, a metal-free, transparent OLED stack reported by Meyer et al.. ${ }^{1}$ where no plasmons can be excited, is investigated for improved outcoupling efficiency.
\end{abstract}

Keywords: organic light-emitting diodes, light extraction, device optimization, quantum efficiency, dipole orientation, transparent OLED

\section{INTRODUCTION}

Organic light-emitting diodes (OLEDs) are promising candidates for lighting applications. Concerning the efficiency of converting electrical power into emitted light, however, they still need to be improved further in order to compete with established lighting technologies. Traditionally, the external quantum efficiency $\eta_{\mathrm{EQE}}$, i.e. the number of photons emitted from the OLED per charge carriers injected into the device, is given by the product of four different factors: ${ }^{2}$

$$
\eta_{\mathrm{EQE}}=\gamma \times \eta_{\mathrm{s} / \mathrm{t}} \times q \times \eta_{\mathrm{out}} .
$$

Therein $\gamma$ is the charge balance factor, $\eta_{\mathrm{s} / \mathrm{t}}$ the singlet/triplet ratio, $q$ the radiative quantum efficiency of the emitter material and $\eta_{\text {out }}$ is the outcoupling factor. As the EQE is desired to be as close to $\eta_{\mathrm{EQE}}=1$ as possible these four factors have to be maximized. It has been shown that $\gamma$ can be brought close to one in heterolayer structures. ${ }^{3}$ For fluorescent emitters one can assume $\eta_{\mathrm{s} / \mathrm{t}}=0.25$ and for phosphorescent emitters $\eta_{\mathrm{s} / \mathrm{t}}=1$. Evidentely, the latter case is preferred. The product $\gamma \times \eta_{\mathrm{s} / \mathrm{t}}$ gives the fraction of injected carriers forming excitons that are in principle able to generate radiation inside an OLED. Their fate is determined by the remaining two factors $q \times \eta_{\text {out }}$. It has been shown that the quantum efficiency $q$ and the outcoupling factor $\eta_{\text {out }}$ are influenced by the OLED stack. As a crude approximation, $\eta_{\text {out }}$ can be estimated as $1 /\left(2 n^{2}\right)$ for isotropic emitters, where $n$ is the refractive index of the emitting organic layer. ${ }^{4}$ However, in detail this value depends on the composition of the OLED stack as well as the position and the orientation of the emitting molecules inside the structure..$^{5,6}$

In this paper, we will use optical simulations to analyze optical loss channels and optimize light-extraction in a well known hetero-layer device with TPD (N,N'-diphenyl-N,N'-bis(3-methylphenyl)-1,1'-biphenyl-4,4-diamine) as hole transporter and $\mathrm{Alq}_{3}$ (tris-(8-hydroxyquinoline) aluminum) as emission and electron transporting layer. We quantify the potential for improved light outcoupling if the orientation of the emitting dipoles can be controlled or if a metal-free, transparent OLED stack is used, where losses by surface plasmon excitation are absent.

Further author information: S.N.: E-mail: stefan.nowy@physik.uni-augsburg.de

W.B.: E-mail: wolfgang.bruetting@physik.uni-augsburg.de 


\begin{tabular}{|lr|}
\hline $\mathrm{Al}$ & $100 \mathrm{~nm}$ \\
\hline $\mathrm{Ca}$ & $15 \mathrm{~nm}$ \\
\hline $\mathrm{Alq}_{3}$ & $80 \mathrm{~nm}$ \\
\hline $\mathrm{TPD}$ & $80 \mathrm{~nm}$ \\
\hline PEDOT:PSS & $30 \mathrm{~nm}$ \\
\hline ITO & $140 \mathrm{~nm}$ \\
\hline glass \\
\hline
\end{tabular}

Figure 1: Bottom-emitting OLED stack with transparent indium tin oxide (ITO) anode and $\mathrm{Alq}_{3}$ as emitter.

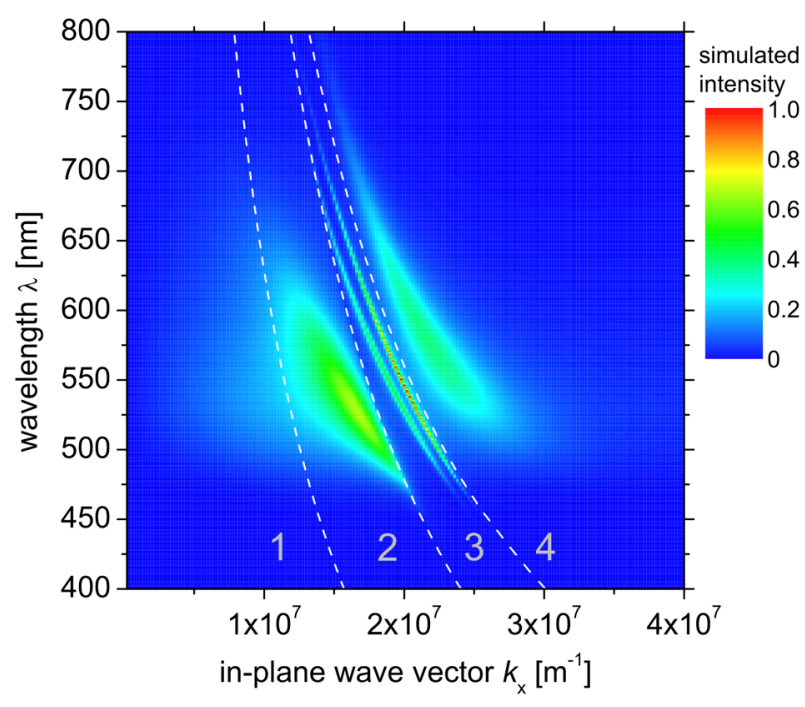

Figure 2: Power dissipation spectrum of an ITO OLED (fig. 1): 1) light emitted directly from the OLED, 2) light trapped inside substrate, 3) waveguided modes, 4) surface plasmon polaritons (SPPs).

\section{SIMULATION METHOD}

The design of OLEDs must consider near field phenomena and the photonic mode density due to the use of thin organic films. ${ }^{6}$ Chance, Prock and Silbey (CPS) ${ }^{7,8}$ adapted a model for radiowaves from Sommerfeld ${ }^{9}$ for molecular fluorescence and energy transfer near interfaces. This model was extended to the near-field optics of OLEDs by various authors, ${ }^{5,6,10-12}$ where an exciton within the OLED is modeled as a point dipole driven by the reflected electromagnetic waves inside a microcavity. The simulations presented in this work are also based on this traditional CPS approach of emissive dipoles. The dipoles, embedded in the multilayer stack of an OLED, are treated as forced damped harmonic oscillators. Their electric field gets refelected at interfaces. The magnitude of this reflected electric field at the dipole position determines the change of decay rate of the emitter, either accelerating or decelerating its emission. A detailed description of the simulation method has been published elsewhere. ${ }^{13}$

All layers in the multilayer stack are treated as infinite in two dimensions, having a certain thickness in the third direction. The interface between two layers is assumed sharp and without roughness. The emission zone (dipole layer) is assumed to be infinitely thin. For an example hetero-layer OLED stack presented in fig. 1 it is located between the hole-transporting layer (TPD) and the electron-transporting layer $\left(\mathrm{Alq}_{3}\right)$. The orientation of the emitting molecules is taken to be isotropic. Furthermore, the radiative quantum efficiency of $\mathrm{Alq}_{3}$ is taken to be $20 \%$, i.e. equal to the PL quantum efficiency.

As a simulation result we obtain a polychromatic power dissipation spectrum (fig. 2), i.e. the contribution of modes with in-plane wave vector component $k_{\mathrm{x}}$ to the spontaneous emission decay rate of the dipoles at each wavelength. We would like to point out that our simulations are polychromatic calculations considering the actual emission spectrum of the dye.

The power dissipation spectrum is split up into four regions. Region $1\left(k_{\mathrm{x}}<\frac{2 \pi}{\lambda} n_{\text {air }}\right)$ represents the light which can leave the OLED directly ('direct emission'). Region $2\left(\frac{2 \pi}{\lambda} n_{\text {air }}<k_{\mathrm{x}}<\frac{2 \pi}{\lambda} n_{\text {glass }}\right)$ denotes the amount of light which is emitted to the glass substrate and is trapped there due to total internal reflection ('emission to substrate'). This fraction is accessible only if outcoupling structures are used. For even larger values of $k_{\mathrm{x}}$ the radiation of the dipoles does no longer reach the glass substrate but is waveguided in the organic layers and the 


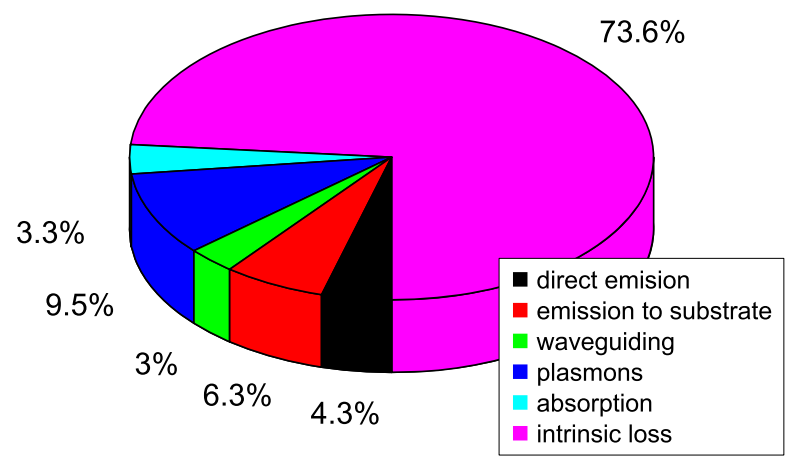

Figure 3: Fraction of power coupled into the different modes for the OLED shown in fig. $1(q=0.2)$.

adjacent ITO electrode ('waveguiding', region 3). Finally, for $k_{\mathrm{x}}>\frac{2 \pi}{\lambda} n_{\mathrm{ITO}+\text { organic }}$, where $n_{\mathrm{ITO}+\text { organic }}$ denotes an effective refractive index of the ITO and organic layers taken together, the emitting dipoles couple to surface plasmon polaritons (SPPs) traveling at the organic-cathode interface ('plasmons', region 4). Integrating over the different regions in the polychromatic power dissipation spectrum leads to the amount of power coupled into the different modes (see fig. 3). In this OLED stack (fig. 1) $4.3 \%$ of the generated light is directly emitted, $6.3 \%$ emitted to the substrate, $3.0 \%$ waveguided, $9.5 \%$ coupled to the plasmons, $3.3 \%$ is absorbed in the stack. The intrinsic loss due to the non-ideal emitter is $73.6 \%$ in this particular OLED stack.

\section{DEVICE OPTIMIZATION}

\subsection{Layer thickness variation and influence of the emitter quantum efficiency $q$}

Optimal layer thicknesses are needed to maximize the light outcoupling from the device. For the OLED presented in fig. 1 we vary the thickness of the $\mathrm{Alq}_{3}$ layer to show its influence on the optical device performance. Fig. 4 shows the power contribution to the different modes calculated from the power dissipation spectra. The emitter quantum efficiency is set to $q=0.2$, determined independently by us. ${ }^{14,15}$ As the dipole layer is moved further away from the cathode the coupling to SPPs is reduced drastically. For the direct emission there exist several local maxima and minima as a function of distance. The first maximum at around $60 \mathrm{~nm} \mathrm{Alq}_{3}$ layer thickness shows the highest fraction of outcoupled light (about 4.5\%). This thickness (which at the same time is the distance of the dipole layer to the cathode) and also the value of the maximum external quantum efficiency of $1.1 \%$ (calculated with $\gamma=1$ and $\eta_{\mathrm{s} / \mathrm{t}}=0.25$ ) is consistent with the optimal thickness of Alq $\mathrm{q}_{3}$ found in experimental work. ${ }^{16-18}$ However, if outcoupling structures are attached to the OLED glass substrate the maximum fraction of totally outcoupled light (about 10.6\%) is achieved with a slightly larger Alq $\mathrm{q}_{3}$ layer thickness of about $80 \mathrm{~nm}$. As a consequence for device optimizations one has to take into account if the device is going to be used with outcoupling structures, as the layer thicknesses for maximum light-outcoupling are different with or without these structures.

A second maximum in the direct emission appears at $230 \mathrm{~nm}$, which is slightly lower in value (about $4.0 \%$ ) than the first maximum. However, if we now assume that $\mathrm{Alq}_{3}$ would be an emitter with quantum efficiency $q=1$ this second maximum is even higher than the first one (fig. 5). This shows that the optimal layer thickness for best device performance is dependent on the quantum efficiency $q$ of the emitter material and has to be considered beforehand in the OLED simulation. If the quantum efficiency is not known, it can be extracted from layer thickness variations and corresponding device simulations, as we have shown recently. ${ }^{19}$

\subsection{Influence of the dipole orientation}

We have shown in the previous section that light outcoupling is improved by choosing optimal layer thicknesses and materials with a high quantum efficiency, preferably $q=1$. However, even then only about $40-50 \%$ of the light generated can be outcoupled (using outcoupling techniques such as lenses or micro-arrays) as can be seen 


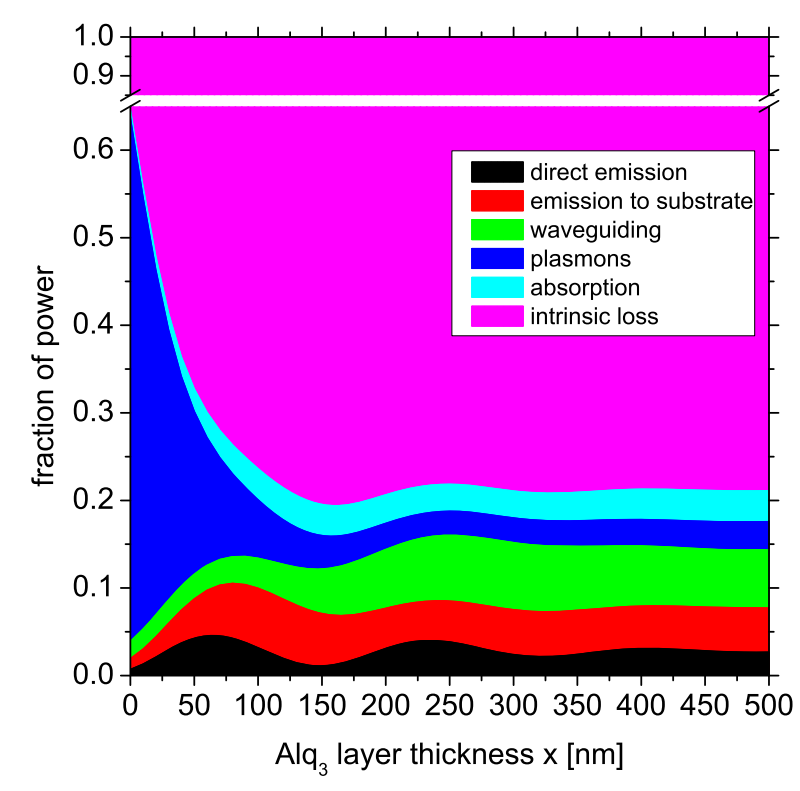

Figure 4: $\mathrm{Alq}_{3}$ layer thickness variation, radiative quantum efficiency $q=0.2, \Delta x=10 \mathrm{~nm}$.

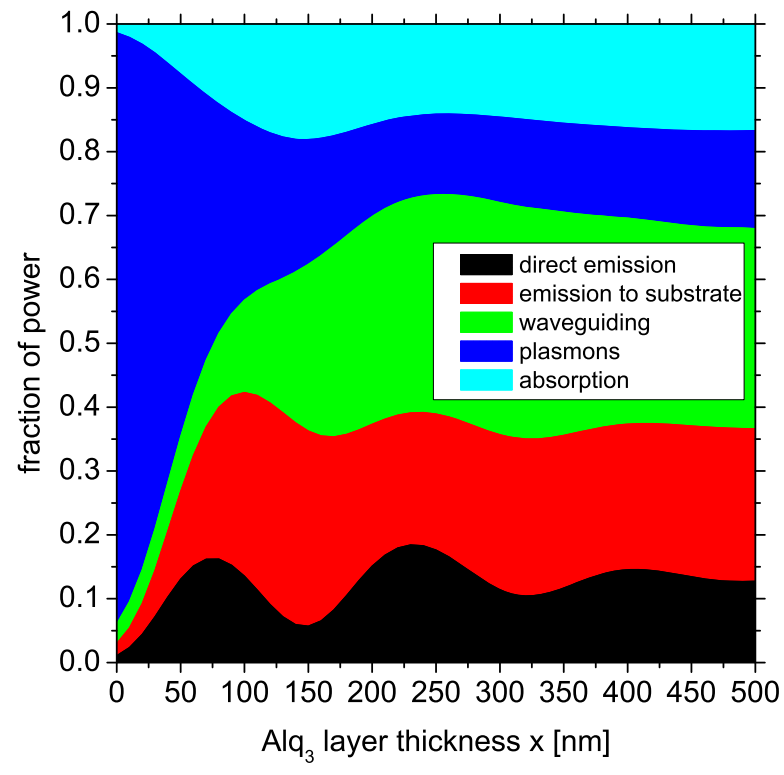

Figure 5: $\mathrm{Alq}_{3}$ layer thickness variation, radiative quantum efficiency $q=1.0, \Delta x=10 \mathrm{~nm}$.

in fig. 5. The remaining light, if not absorbed, either is waveguided or excites plasmons at the metallic cathode. To further improve OLEDs other techniques have to be considered.

In our simulations the isotropic emission of the dipoles is a mixture of three dipole orientations: one dipole perpendicular and two dipoles parallel to the interfaces of the OLED (fig. 6a). For the OLED presented in fig. 1 the power dissipation spectra for each dipole orientation is shown in fig. 6 . The total power dissipation spectrum, already shown in fig. 2, is composed of these three dipole orientations. The perpendicular dipole mainly is responsible for the excitation of the plasmon (fig. 6b). One orientation of the parallel dipoles couples to the TE waveguiding mode, to a large fraction of light trapped in the substrate, and to light emitted directly (fig. 6c). The other parallel dipole couples to the TM waveguiding mode, to light emitted directly or trapped in the substrate, and slightly contributes to the plasmon (fig. 6d).

As the coupling to the plasmon is undesired for high performance OLEDs it would be best if this mode would not be excited at all. From fig. 6 one concludes that if the perpendicular dipole orientation is avoided one could reach better outcoupling efficiencies. Simulation where only the parallel dipole orientations are considered shows that the direct emission is increased from $16.2 \%$ to $23.0 \%$ for the standard OLED stack of fig. 1 with $q=1$ (fraction of power for the different modes see fig. 7 for isotropic dipole orientation and fig. 8 for parallel dipole orientation). If outcoupling structures are used the increase is from $40.0 \%$ to $56.5 \%$, which is an enhancement of about $41 \%$. The coupling to the plasmons is reduced from $35.9 \%$ to $14.9 \%$. Further optimizations, e.g. layer thickness variations, might even yield higher outcoupling values. However, controlling the dipole orientation during fabrication of the OLED is a major issue, but it has been shown in literature that ordered dipole moments can be achieved in devices. ${ }^{20-23}$

\subsection{Transparent OLED without metal electrodes}

In the previous sections we have seen that OLED performance can be enhanced with optimal layer thicknesses and reducing the coupling to plasmons by "choosing" parallel dipole moment orientations. Another possibility to avoid losses due to plasmons is to use metal-free, transparent OLED structures. Such a system has been published recently by Meyer et al. ${ }^{1}$ It consists of a glass substrate, $130 \mathrm{~nm}$ ITO, $40 \mathrm{~nm}$ BPhen:Li, $5 \mathrm{~nm}$ TPBi, $15 \mathrm{~nm}$ TPBi:Ir(ppy $)_{3}, 40 \mathrm{~nm}$ TCTA, $\mathrm{WO}_{3}$ with variable thickness $(20-80 \mathrm{~nm})$, and $60 \mathrm{~nm}$ of sputtered ITO. The $\mathrm{WO}_{3}$ layer acts as buffer layers to protect the organic layers from the sputter process. They find that the 


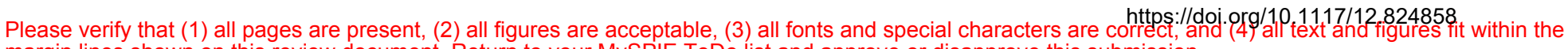

margin lines shown on this review document. Return to your MySPIE ToDo list and approve or disapprove this submission.
Organic Light Emitting Materials and Devices XIII : 2-6 August 2009; San Diego, California, United States, S. 74151C

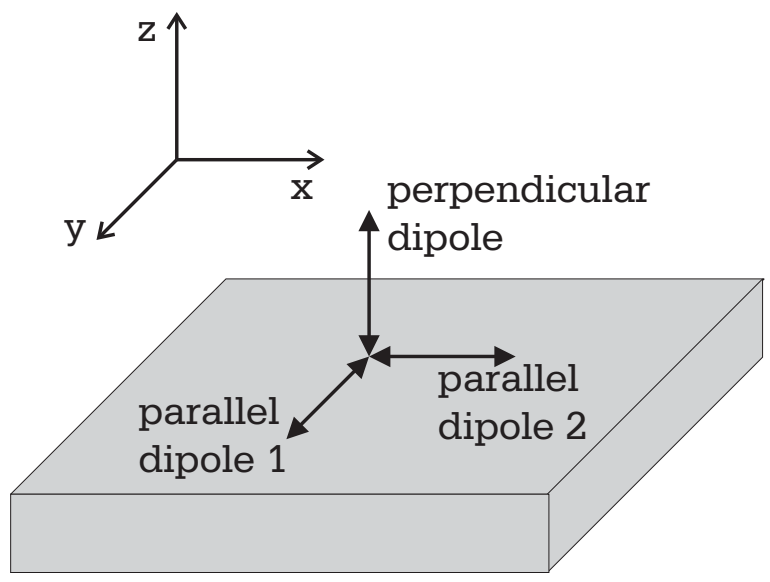

(a) Dipole orientations used in the simulations.

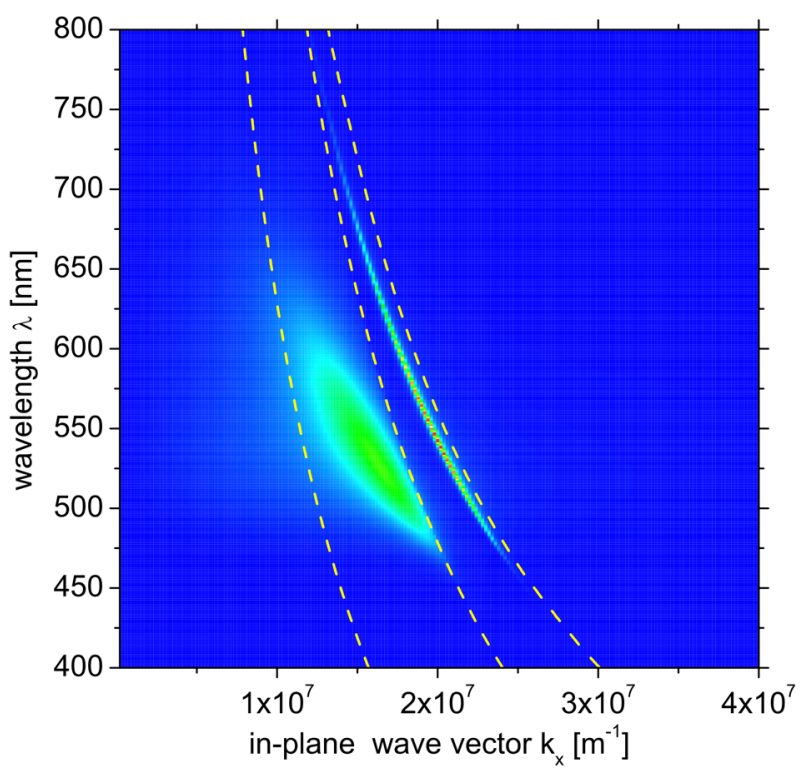

(c) Parallel dipole orientation 1 .

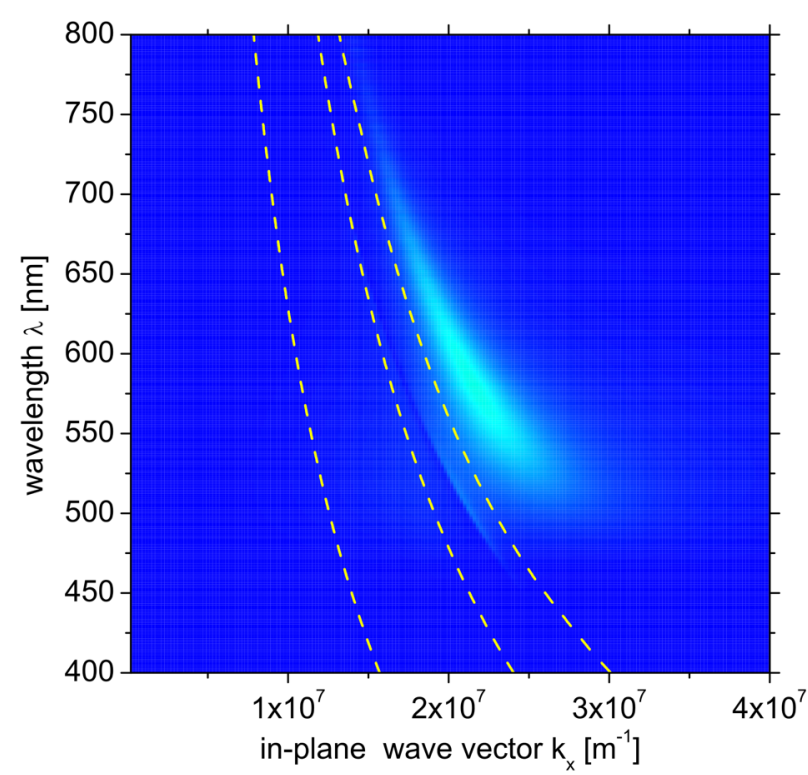

(b) Perpendicular dipole orientation.

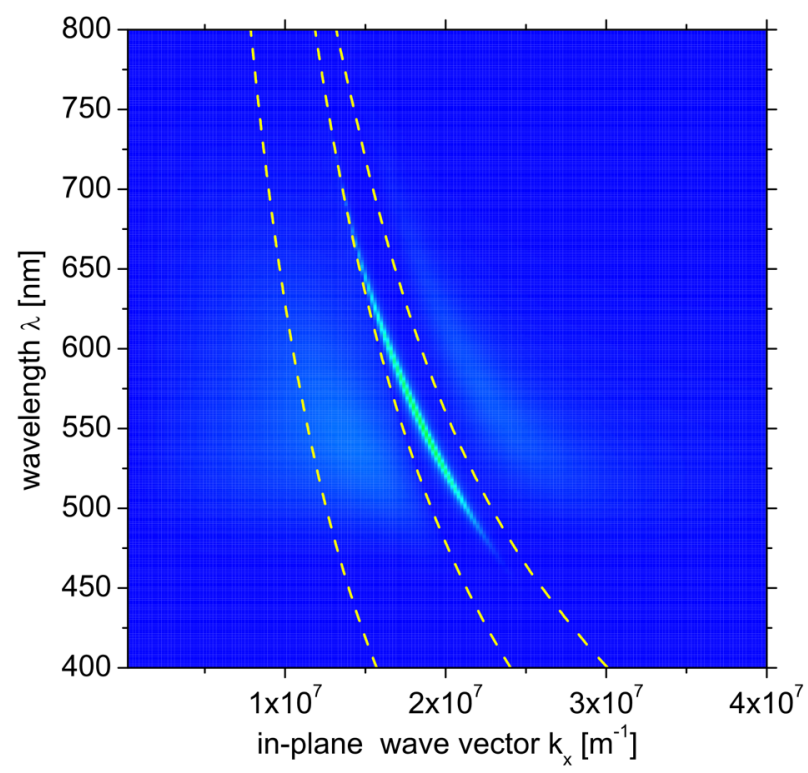

(d) Parallel dipole orientation 2.

Figure 6: Power dissipation spectra for different dipole orientations of the OLED presented in fig. 1. All spectra normalized to the same factor. 


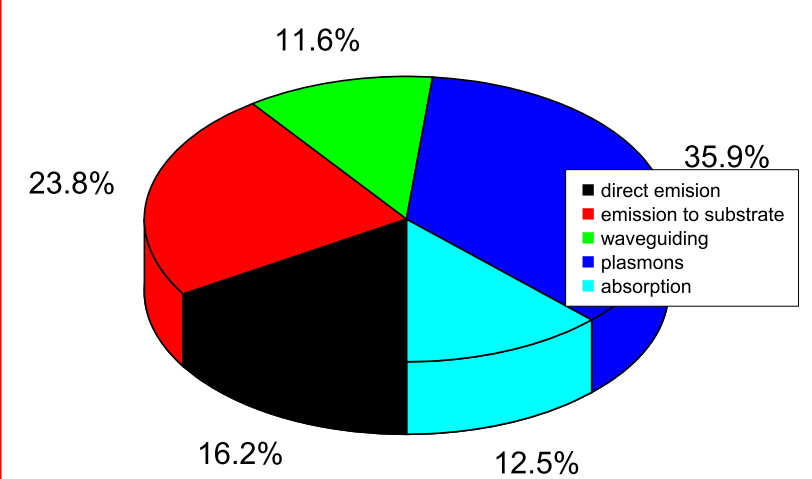

Figure 7: Fraction of power coupled into the different modes for the OLED shown in fig. $1(q=1)$. Isotropic dipole orientation.

\begin{tabular}{|lr|}
\hline ITO & $60 \mathrm{~nm}$ \\
\hline $\mathrm{WO}_{3}$ & $\mathrm{x} \mathrm{nm}$ \\
\hline TCTA & $40 \mathrm{~nm}$ \\
\hline TPBi:Ir(ppy $)_{3}$ & $15 \mathrm{~nm}$ \\
\hline TPBi & $5 \mathrm{~nm}$ \\
\hline BPhen:Li & $40 \mathrm{~nm}$ \\
\hline ITO & $130 \mathrm{~nm}$ \\
\hline glass \\
\hline
\end{tabular}

Figure 9: Transparent OLED stack used by Meyer et $a .^{1}$

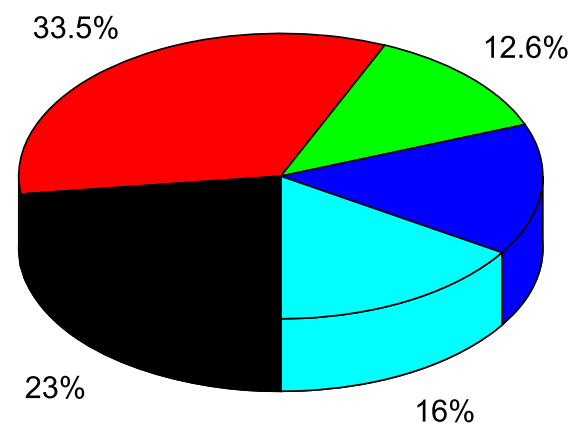

$14.9 \%$

Figure 8: Fraction of power coupled into the different modes for the OLED shown in fig. $1(q=1)$. Only parallel dipole orientation.

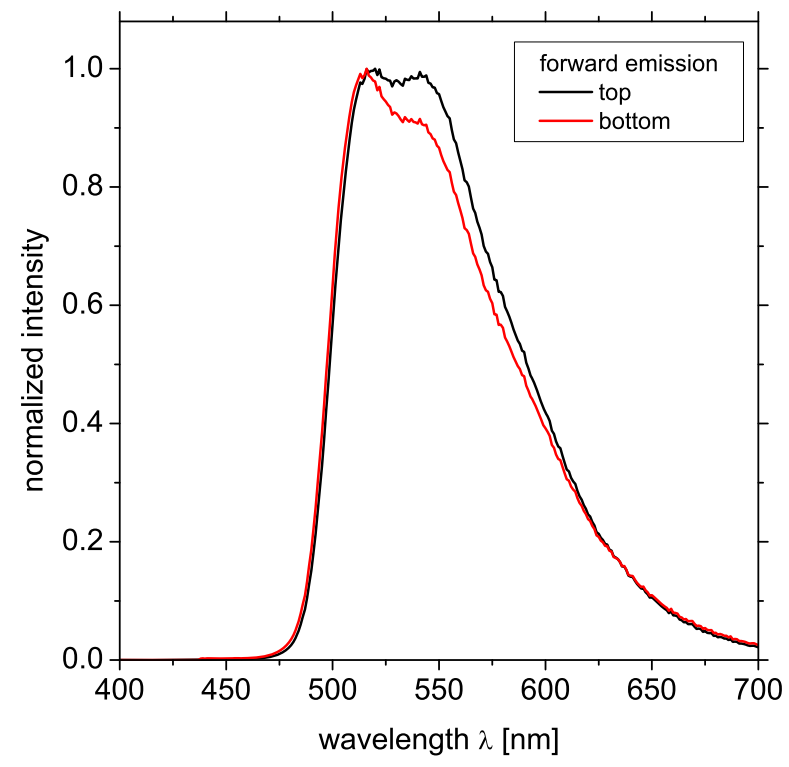

Figure 10: Simulated top (black curve) and bottom (red curve) emission in forward direction of the OLED presented in fig. 9 with $60 \mathrm{~nm} \mathrm{WO}_{3}$ layer thickness.

optimal thickness of the buffer layer is around $60 \mathrm{~nm}$ in terms of protection, as well as current efficiency and power efficiency. In the following, we analyze this OLED stack with our optical simulation tool. We assume a quantum efficiency of $q=1$ for the $\operatorname{Ir}(\text { ppy) })_{3}$. However, this value might be lower in reality, which then would change the simulation results slightly, as has been shown above for the TPD/Alq ${ }_{3}$ OLED.

As there are no reflecting electrodes the device is transparent and emits light on both sides. The emission pattern in bottom (glass side) and top direction is shown in fig. 10 for a device with $60 \mathrm{~nm} \mathrm{WO}_{3}$. The two spectra agree well with the data published by Meyer et al. ${ }^{1}$ Experimentally it was found that nearly $70 \%$ of the total outcoupled light is emitted through the bottom side. Our simulation yields a value of $60 \%$ for this $60 \mathrm{~nm}$ $\mathrm{WO}_{3}$ device (fig. 11). The difference is that the simulation considers the total half-space into which the OLED emits its light, whereas the measurement is done only in the direction perpendicular to the device. 


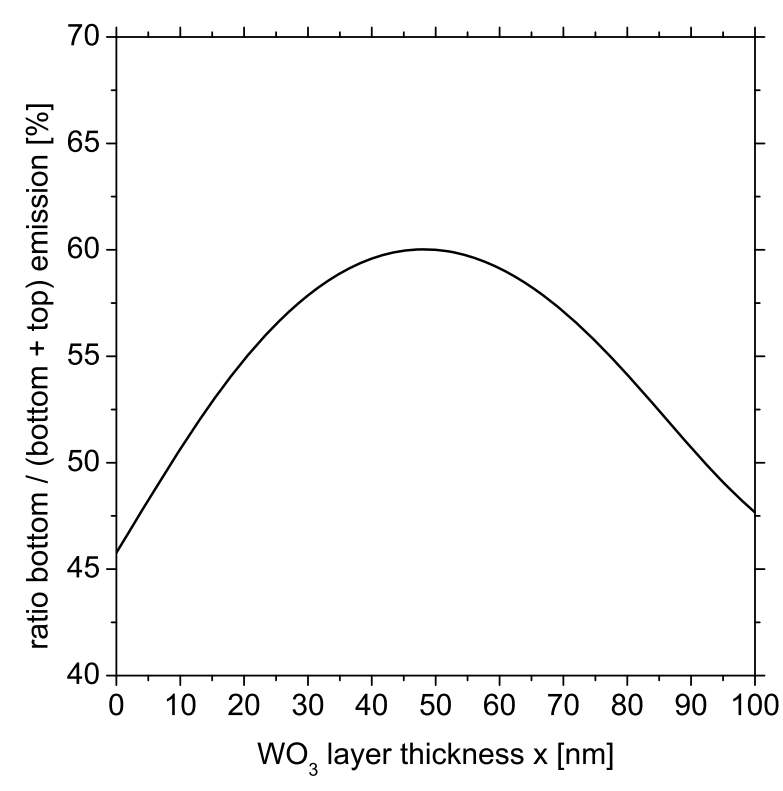

Figure 11: Ratio of the bottom and total light emission for different $\mathrm{WO}_{3}$ layer thickness. $\Delta x=2 \mathrm{~nm}$.

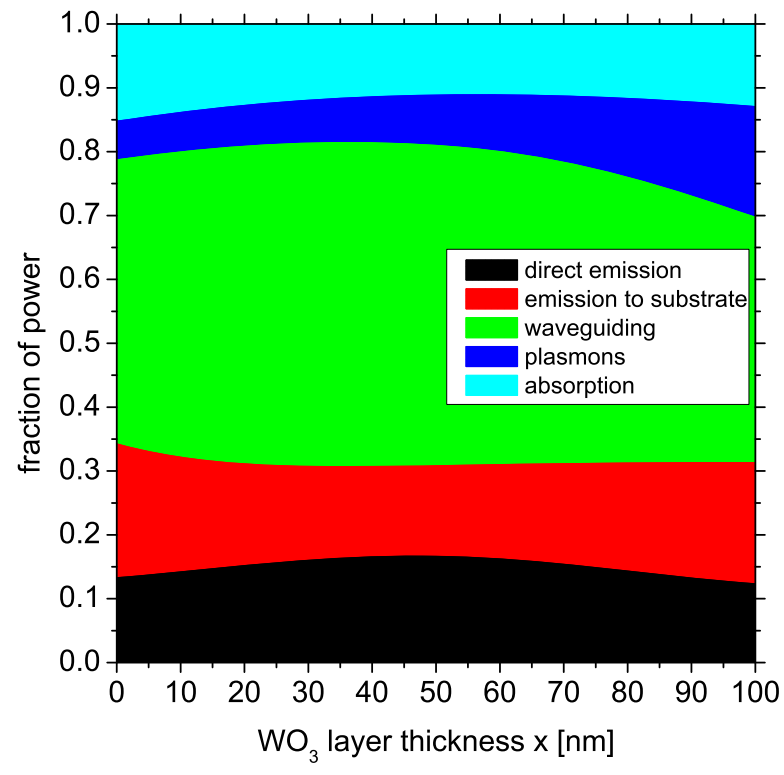

Figure 12: Power coupled into the different modes for different $\mathrm{WO}_{3}$ layer thickness. $\Delta x=2 \mathrm{~nm}$.

Now the $\mathrm{WO}_{3}$ layer thickness is varied and the amount of power coupled into the different modes is analyzed (see fig. 12). For light emitted directly there exists a broad maximum at about $46 \mathrm{~nm} \mathrm{WO}_{3}$ thickness of $16.6 \%$. However, the optimal layer thickness has been reported to be around $60 \mathrm{~nm}$. This might be related to the use of $\mathrm{WO}_{3}$ as a buffer layer in the ITO sputter process: thin layers $(<60 \mathrm{~nm})$ do not protect the OLED sufficiently, hence the OLED is damaged and light output is decreased. As the OLED is metal-free one expects that no plasmons are excited. If the emitting dipoles do not couple energy into plasmons this "free" amount of energy could instead increase light outcoupling. But fig. 12 shows that even if an outcoupling structure is used on the glass substrate side only about $35 \%$ of the generated light is emitted from the OLED. This is even less as for the device with conventional metal cathode shown earlier (fig. 5). The reason is that a huge amount of power is coupled into waveguiding modes*. As the amount of power coupled to total light emission and absorption is similar to the device of fig. 5 one concludes that the power distribution is just shifted from the plasmons to the waveguiding modes for the transparent OLED. This means that a rise in outcoupled power is not achieved by substituting the metal electrodes with transparent ones per se. Other outcoupling techniques, like scattering out waveguiding modes or plasmons, and device concepts have to be developed for devices which can emit more than $40 \%$ of the light generated.

\section{SUMMARY}

In conclusion the simulations presented here show that the dipole layer has to be located at the right position, so that the feedback exerted by the cavity on the emitting molecules is optimal for the fraction of light emitted directly or to the glass substrate and the fraction coupled to waveguided modes or SPPs is minimized. An important point is that the optimal dipole layer position (and thus the device structure) also depends on the radiative quantum efficiency $q$ of the material used as emitter. We have shown that the orientation of the dipole moment could improve the amount of outcoupled light significantly. Furthermore, we have shown on a metal-free, transparent OLED stack that the coupling to plasmons can be reduced drastically, unfortunately to the benefit of waveguiding modes and does not necessarily lead to improved outcoupling of light.

${ }^{*}$ The plasmon contribution in this simulation is not expected due to the metal-free electrodes. Analysis of the power dissipation spectra shows that the waveguiding modes "leak" into the $k_{\mathrm{x}}$ region where plasmons are excited. 


\section{ACKNOWLEDGMENTS}

The authors would like to thank the German Federal Ministry of Education and Research (BMBF) for funding part of this work under contract FKZ 13N10474 (TOPAS). We would like to thank Sami Hamwi (TU Braunschweig, Germany) for optical constants of their transparent OLED system.

\section{REFERENCES}

[1] Meyer, J., Winkler, T., Hamwi, S., Schmale, S., Johannes, H.-H., Weimann, T., Hinze, P., Kowalsky, W., and Riedl, T., "Transparent inverted organic light-emitting diodes with a tungsten oxide buffer layer," Advanced Materials 20, 3839-3843 (2008).

[2] Tsutsui, T., Aminaka, E., Lin, C. P., and Kim, D.-U., "Extended molecular design concept of molecular materials for electroluminescence: sublimed-dye films, molecularly doped polymers and polymers with chromophores," Philosophical Transactions of the Royal Society of London A 355, 801 - 814 (1997).

[3] Tang, C. W. and VanSlyke, S. A., "Organic electroluminescent diodes," Applied Physics Letters 51, 913 915 (September 1987).

[4] Greenham, N. C., Friend, R. H., and Bradley, D. D. C., "Angular dependence of the emission from a conjugated polymer light-emitting diode: Implications for efficiency calculations," Advanced Materials 6(6), 491 - 494 (1994).

[5] Novotny, L., "Allowed and forbidden light in near-field optics. I. A single dipolar light source," Journal of the Optical Society of America A 14(1), 91 - 104 (1997).

[6] Barnes, W. L., "Fluorescence near interfaces: the role of photonic mode density," Journal of Modern Optics 45(4), 661 - 699 (1998).

[7] Chance, R. R., Prock, A., and Silbey, R., "Lifetime of an emitting molecule near a partially reflecting surface," The Journal of Chemical Physics 60(7), 2744 - 2748 (1974).

[8] Chance, R. R., Prock, A., and Silbey, R., "Molecular fluorescence and energy transfer near interfaces," Advances in Chemical Physics 37, 1-65 (1978).

[9] Sommerfeld, A., "Über die Ausbreitung der Wellen in der drahtlosen Telegraphie," Annalen der Physik 28(4), 665 - 736 (1909).

[10] Wasey, J. A. E. and Barnes, W. L., "Efficiency of spontaneous emission from planar microcavities," Journal of Modern Optics 47(4), 725 - 741 (2000).

[11] Smith, L. H., Wasey, J. A. E., Samuel, I. D. W., and Barnes, W. L., "Light out-coupling efficiencies of organic light-emitting diode structures and the effect of photoluminescence quantum yield," Advanced Functional Materials 15(11), 1839 - 1844 (2005).

[12] Celebi, K., Heidel, T. D., and Baldo, M. A., "Simplified calculation of dipole energy transport in a multilayer stack using dyadic green's functions," Optics Express 15(4), 1762 - 1772 (2007).

[13] Nowy, S., Krummacher, B. C., Frischeisen, J., Reinke, N. A., and Brütting, W., "Light extraction and optical loss mechanisms in organic light-emitting diodes: Influence of the emitter quantum efficiency," Journal of Applied Physics 104, 123109 (2008).

[14] Cölle, M., Gmeiner, J., Milius, W., Hillebrecht, H., and Brütting, W., "Preparation and charecterization of blue-luminescent tris(8-hydroxyquinoline)aluminum $\left(\mathrm{Alq}_{3}\right)$," Advanced Functional Materials 13(2), 108 - 112 (2003).

[15] Cölle, M. and Brütting, W., "Thermal, structural and photophysical properties of the organic semiconductor $\mathrm{Alq}_{3}, "$ Physica Status Solidi A 201(6), 1095 - 1115 (2004).

[16] So, S. K., Choi, W. K., Leung, L. M., and Neyts, K., "Interference effects in bilayer organic light-emitting diodes," Applied Physics Letters 74(14), 1939 - 1941 (1999).

[17] Schmitz, C., Thelakkat, M., and Schmidt, H.-W., "A combinatorial study of the dependence of organic LED characteristics on layer thickness," Advanced Materials 11(10), 821 - 826 (1999).

[18] Beierlein, T. A., Combinatorial Methods for Organic Light-Emitting Materials and Devices, PhD thesis, University of Bayreuth, Germany (2003).

[19] Krummacher, B. C., Nowy, S., Frischeisen, J., Klein, M., and Brütting, W., "Efficiency analysis of organic light-emitting diodes based on optical simulation," Organic Electronics 10(3), 478-485 (2009). 
[20] Becker, H., Burns, S. E., and Friend, R. H., "Effect of metal films on the photoluminescence and electroluminescence of conjugated polymers," Physical Review B 56(4), 1893 - 1905 (1997).

[21] Lin, H.-W., Lin, C.-L., Chang, H.-H., Lin, Y.-T., Wu, C.-C., Chen, Y.-M., Chen, R.-T., Chien, Y.-Y., and Wong, K.-T., "Anisotropic optical properties and molecular orientation in vacuum-deposited ter $(9,9-$ diarylfluorene)s thin films using spectroscopic ellipsometry," Journal of Applied Physics 95(3), 881 - 886 (2004).

[22] Lin, H.-W., Lin, C.-L., Wu, C.-C., Chao, T.-C., and Wong, K.-T., "Influences of molecular orientations on stimulated emission charachteristics of oligofluorene films," Organic Electronics 8, 189 - 197 (2007).

[23] Yokoyama, D., Sakaguchi, A., Suzuki, M., and Adachi, C., "Horizontal orientation of linear-shaped organic molecules having bulky substituents in neat and doped vacuum-deposited amorphous films," Organic Electronics 10, 127 - 137 (2009). 